\section{Dementia

\title{
Long-Term Occupational Sleep Loss and Post-Retirement Cognitive Decline or Dementia
}

\author{
Jana Thomas ${ }^{a-c}$ Sebastiaan Overeem ${ }^{d,}$ e Jurgen A.H.R. Claassen ${ }^{a-c}$ \\ ${ }^{a}$ Department of Geriatric Medicine, Radboud University Medical Center, Nijmegen, \\ The Netherlands; ${ }^{b}$ Radboud Alzheimer Center, Nijmegen, The Netherlands; \\ 'Donders Institute for Brain, Cognition and Behavior, Nijmegen, The Netherlands; \\ ${ }^{d}$ Sleep Medicine Center Kempenhaeghe, Heeze, The Netherlands; ${ }^{e}$ Eindhoven University \\ of Technology, Eindhoven, The Netherlands
}

\section{Keywords}

Ageing and cognition · Alzheimer's disease · Cognitive decline · Sleep loss · Shift work

\begin{abstract}
Introduction: Recent evidence suggests that poor sleep is a risk factor that contributes to the development of Alzheimer's disease (AD). Most studies have focused on short-term effects of sleep deprivation on cognitive function, whereas longitudinal studies are limited to self-reported sleep and the risk of later-life dementia. Because sleep loss could be an early manifestation of neurodegenerative disease, reverse causality in these studies cannot be excluded. Objective: In this explorative, observational study, we investigated the effects of extended periods of extrinsically (work-related) caused sleep loss on later-life cognitive function, early dementia symptoms, and current sleep quality. Methods: We approached a community of retired male maritime pilots (approx. $n=500$ ) through a newsletter. We investigated 50 respondents (mean age 71.7 years \pm 7.7 ), with a history of $>25$ years of work on irregular schedules, which resulted in extended periods of sleep loss. Validated questionnaires on cognitive complaints (Cognitive Failure Questionnaire [CFQ]), early dementia symptoms (Early Dementia Questionnaire [EDQ]), current sleep quality (Pittsburgh Sleep Quality Index [PSQI] and sleep-wake diaries), quality of life (QoL, EQ-5D), and mood (Hospital Anxiety and Depression Scale [HADS]) were administered by a single investigator (J.T.), who also completed an observer rating of cognitive function. Results: Scores on the CFQ, EDQ, PSQI, EQ-5D, and HADS were within normal ranges adjusted for age, sex, and education. The observer rating was not indicative of cognitive decline. Conclusion: We found no evidence that long-term exposure to work-related sleep loss had resulted in cognitive decline or early dementia symptoms in this sample of retired maritime pilots.


Thomas et al.: Long-Term Occupational Sleep Loss and Post-Retirement Cognitive Decline

\section{Introduction}

Recently, the relationship between poor sleep and Alzheimer's disease (AD) has gained scientific interest. Studies revealed that AD affects areas in the brain responsible for sleepwake regulations $[1,2]$. Consequentially, $44 \%$ of people with AD suffer from sleep disturbances [3]. These studies found that poor sleep may not only be a symptom of the disease, but also a risk factor for its development and may contribute to its manifestation $[2,4,5]$. One of the major contributors to the neurodegeneration in $\mathrm{AD}$ patients is the accumulation of amyloid-beta $(A \beta)$ proteins in the brain [6].

The cause(s) that drive this accumulation remain uncertain. One hypothesis linking sleep to AD states that amyloid is the main waste product that accumulates during wakefulness due to synaptic activity [7]. During sleep, however, reduced synaptic activity results in a lower production of $A \beta$ [7]. Another hypothesis suggests that a glymphatic system is responsible for the elimination of soluble waste products from the central nervous system, thereby cleaning the brain from waste products during sleep. Loss of sleep could lead to a reduced glymphatic clearance of $A \beta$ [8]. Through either of these hypotheses, poor sleep could increase the risk of developing AD by influencing the production and/or clearance of $A \beta$. Recent studies support this hypothesis. It was found that 1 night of full sleep deprivation increases $A \beta$ levels in cerebrospinal fluid (CSF) by approximately $10 \%$ [9], increases plasma $A \beta$ [10], and increases the accumulation of $A \beta$ in the brain measured with PET-CT [11]. Evidence suggests that sleep affects tau, the second pathological hallmark of AD, in a similar manner as $A \beta$ [12]. In addition, vascular disease may play a role in determining the risk of late-life $\mathrm{AD}$ [13]. Because poor sleep increases cardiovascular risk, this may be a third pathway through which sleep contributes to AD.

The studies reported above looked at the short-term effects on biomarkers of AD. We initiated the BATAVIA (Being Awake Throughout Adulthood affects (cognitive) Vitality In Aging) study to investigate the effects of exposure to extended periods of work-related sleep loss on postretirement cognitive function, dementia symptoms, and sleep quality. Furthermore, we explored the effects of work-related sleep loss on quality of life (QoL) and mood. We recruited a cohort of retired maritime pilots, who had been exposed to extended periods of sleep loss due to irregular and unpredictable work schedules. Sleep loss is characterized as a combination of sleep deprivation (missing a full night of sleep due to work), sleep restriction (a shorter night of sleep), and sleep fragmentation or disruption (short sleep periods interrupted by calls to work). We will refer to these under the umbrella term "sleep loss" throughout.

We hypothesized that this group is at an increased risk of developing cognitive impairment or dementia and that current sleep quality may be disturbed after long-term chronic sleep loss due to circadian rhythm distortion. These relationships could be more pronounced in participants that have been exposed for a longer time (greater amount of work years) and less pronounced in participants with a longer recovery time (greater amount of retirement years).

\section{Materials and Methods}

Design and Procedure

The BATAVIA study was an explorative study, investigating the effect of prolonged work-related sleep loss on cognitive decline, early signs of dementia, and sleep quality. Participants were scheduled for 1 visit at which they supplied answers to 6 questionnaires. 
Thomas et al.: Long-Term Occupational Sleep Loss and Post-Retirement Cognitive Decline

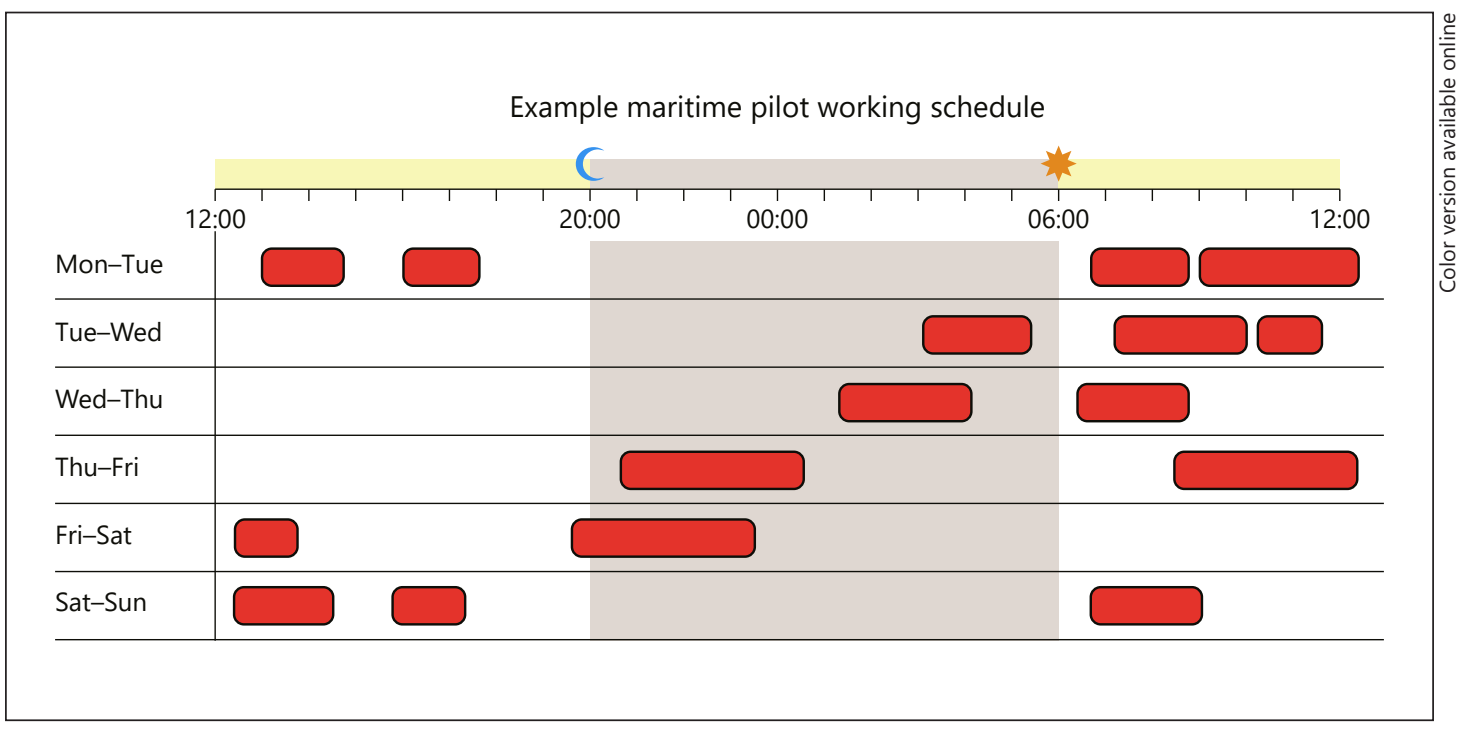

Fig. 1. Example of a maritime pilot's work schedule. Working hours are indicated in red (dark grey). This figure was presented previously in another of our publications [18].

\section{Participants}

We recruited 50 retired male maritime pilots with work-related sleep loss. Details about the study population and recruitment can be found in the online supplementary material (see www.karger.com/ doi/10.1159/000504020). An example of a typical work week is illustrated in Figure 1.

\section{Measures}

A questionnaire about health, medication use, medical history, and the number of work years and retirement years was created. We further used a series of well-validated and sensitive questionnaires to collect information about subjective cognitive decline (Cognitive Failure Questionnaire [CFQ]), early dementia symptoms (Early Dementia Questionnaire [EDQ]), current sleep quality (Pittsburgh Sleep Quality Index [PSQI] and sleep-wake diaries), QoL (EQ-5D), and mood (Hospital Anxiety and Depression Scale [HADS]). Detailed information about the questionnaires is summarized in the online supplementary material. Because all questionnaires were answered in a self-reporting manner, an observer rating was completed (OPS) by the investigator with remarks on orientation, practical skills, and social skills, to assess overall cognitive function of participants.

\section{Statistical Analysis}

Analyses were performed using IBM SPSS (SPSS Inc., Chicago, IL, USA). The $\alpha$ value was set at $p<0.05$ and tests were conducted two-sided. Outcomes are reported as means and standard deviation ( \pm SD). Correlations were performed using Pearson's correlation coefficient. Correlations of $r<0.3$ were interpreted as no/weak correlation and were negligible. Only correlations of $r>0.3$ are reported. Significant correlations are reported with 95\% confidence intervals (CI). Confounders were addressed with partial correlation analyses.

\section{Results}

Fifty participants completed the study. Of these, 3 completed them digitally as they lived abroad. No participants were on medication known to influence cognition or sleep, including sleep medication or antidepressants. Baseline characteristics are presented in Table 1. 
Dementia

and Geriatric

Cognitive Disorders

Table 1. Baseline characteristics of 50 retired maritime pilots

\begin{tabular}{l|l}
\hline Dement Geriatr Cogn Disord 2019;48:105-112 \\
\hline DOI: 10.1159/000504020 & $\begin{array}{l}\text { @ 2019 The Author(s). Published by S. Karger AG, Basel } \\
\text { www.karger.com/dem }\end{array}$ \\
\hline
\end{tabular}

Thomas et al.: Long-Term Occupational Sleep Loss and Post-Retirement Cognitive Decline

\begin{tabular}{lc}
\hline Age, years & $71.7 \pm 7.7$ \\
BMI & $26.3 \pm 3.5$ \\
Number of working years & $26 \pm 3$ \\
Number of retirement years & $16 \pm 8.4$ \\
A history of diabetes & $0(0)$ \\
Medication (yes/no) & $38(76)$ \\
Smoking & $1(2)$ \\
A history of hypertension & $18(36)$ \\
A history of high cholesterol & $13(26)$ \\
Living with family & $44(88)$ \\
Living alone & $24(12)$ \\
Married & $40(80)$ \\
Widower & $4(8)$
\end{tabular}

Values are expressed as mean \pm SD or $n(\%)$.

Table 2. Results for subjective cognitive functioning assessed with the Cognitive Failure Questionnaire (CFQ)

\begin{tabular}{llrcc}
\hline Measure & Minimum & Maximum & Mean \pm SD & $\begin{array}{l}\text { Norm values established by } \\
\text { Ponds et al. [14], 2006 }\end{array}$ \\
\hline CFQ Confusion & 0 & 15 & $6.0 \pm 3.2$ & $7.7 \pm 3.6$ \\
CFQ Social Confusion & 1 & 9 & $5.0 \pm 2.1$ & $5.7 \pm 2.4$ \\
CFQ Names and Words & 1 & 10 & $5.2 \pm 2.1$ & $5.6 \pm 2.1$ \\
CFQ Orientation & 0 & 4 & $1.6 \pm 1.3$ & $3.0 \pm 1.9$ \\
CFQ overall score & 7 & 50 & $24.6 \pm 10.0$ & $31.8 \pm 11.1$ \\
\hline
\end{tabular}

\section{Subjective Cognitive Functioning (CFQ)}

All scores on the CFQ were within the normal range. The highest sub-score was observed on the confusion subscale and the lowest on the orientation subscale. We compared our results to the norms established by Ponds et al. [14] from the MAAS study (Table 2). No significant correlations were found between the number of working years (exposure to sleep disruptions) and CFQ scores.

\section{Early Dementia Questionnaire (EDQ)}

With 0 as the lowest median score and 3.5 as the highest, all participants remained below the cut-off median of 8, which indicates that participants don't show signs of early dementia. The overall mean score was $1.6 \pm 1.2$. The highest score on the EDQ was observed on the memory subscale (mean $6.0 \pm 2$ ). Concentration was the second highest score (mean $2.3 \pm$ 1.9), followed by emotional issues (mean $1.6 \pm 1.5$ ), and sleep problems (mean $1.5 \pm 1.6$ ). Pearson's correlation analysis indicated positive correlations between the number of years retired and physical issues $(r=0.51 ; p<0.001 ; 95 \%$ CI $0.39-0.66)$ and the EDQ overall score $(r=0.3 ; p=0.03 ; 95 \% \mathrm{CI} 0.02-0.55)$. However, when controlling for age as possible confounder, partial correlations remained insignificant. No significant correlation was found for the number of working years.

\section{Sleep: PSQI and Sleep-Wake Diary}

Participants had a mean PSQI of $4.0 \pm 2.6$, which is below the cut-off of 5, indicating that they had no sleep problems when examining the overall mean. Regarding sleep disturbances, $66 \%(n=33)$ scored below, and 34\% (n=17) scored above the cut-off, with the highest score 
Thomas et al.: Long-Term Occupational Sleep Loss and Post-Retirement Cognitive Decline

of 15 , reported by 1 participant. However, no significant correlation was found between the number of working years and the PSQI, or between the number of years retired (time of recovery) and the PSQI.

Participants maintained a sleep-wake diary for 10 days. A mean was calculated for the 3 most relevant variables: sleep onset latency (SOL), total sleep time (TST), and number of awakenings. Overall, participants did not show anomalies, with a mean SOL of $18 \pm 19 \mathrm{~min}$, a mean TST of 7:09 $\mathrm{h} \pm 00: 43$, and a mean of $2 \pm 0.9$ awakenings per night. Furthermore, no differences were observed when comparing week-to-weekend rhythms, concerning bedtimes and wake-up times. The daily intraindividual variation in bedtimes and wake-up times was also not different; apart from some deviation, most participants did not differ $>2 \mathrm{~h}$ in bedtimes and wake-up times. Correlation analyses did not indicate any association between the amount of working years or years retired and the 3 main sleep-wake diary entries.

\section{QoL (EQ-5D) and Mood (HADS)}

With a mean of $<2$ on each subscale, all participants indicated that they experience a good QoL. This was further confirmed by a mean utility score of $0.9 \pm 0.1$ and a visual analog scale (VAS) score of $81.9 \pm 13.1$. Slight problems regarding mobility and pain were the most frequent QoL issues, whereas problems regarding self-care were the least frequent. Correlation analysis indicated a significant positive correlation between the number of years retired and the overall score on the EQ-5D ( $r=0.4 ; p=0.001 ; 95 \%$ CI $0.05-0.64)$, and a negative correlation between the number of years retired and the utility score $(r=-0.4 ; p=0.004 ; 95 \% \mathrm{CI}$ -0.6 to -0.25$)$. Again, these correlations were mediated by age, as confirmed by partial correlation analysis. No correlation was found between the amount of working years and the EQ-5D.

None of the participants had a HADS score above the cut-off. Concerning anxiety, 48 participants (96\%) scored within the normal range of 0-7 (mean $2.7 \pm 2.2$ ) and regarding depression, 46 participants (92\%) scored within the normal range of 0-7 (mean 2.9 \pm 2.4 ).

\section{Observer Rating (OPS)}

The overall mean OPS score was $24.9 \pm 2.2$, suggesting no indications of cognitive dysfunction.

\section{Discussion}

We explored the effects of extended exposure to work-related sleep loss on later-life cognitive health and sleep quality. There were no correlations between exposure to sleep loss and cognitive complaints or indications for early-stage dementia, nor did we find negative effects on mood or QoL. Initially, we hypothesized that longer exposure to, and longer recovery from work-related sleep loss impacts the prevalence of cognitive complaints. However, with regard to cognitive functioning, all participants scored normally. We detected a broad variation among CFQ scores, with the lowest score (indicating best performance) on the orientation subscale, probably related to their former profession as maritime pilots that required orientation/navigational skills. From the literature, it is known that approximately $8 \%$ of adults aged $>65$ years suffer from dementia, for people aged $>80$ years the prevalence is $25 \%$. Moreover, approximately $10-20 \%$ of people aged $>65$ years have mild cognitive impairment (MCI). Based on this prevalence in the general population, our sample could have had an estimated 5-15 participants with MCI or dementia. Since our participants were chronically exposed to sleep loss, we hypothesized that the prevalence of dementia would be higher than in the general population. However, with regard to early signs of dementia, participants 
Thomas et al.: Long-Term Occupational Sleep Loss and Post-Retirement Cognitive Decline

scored within the normal ranges and no relationship was found between the amount of exposure to sleep loss and early signs of dementia.

We discovered an association between the amount of retirement years and the EDQ which was mediated by age. This indicates that older age correlates with physical issues and issues regarding memory, concentration, mood, and sleep. Regarding sleep, we expected that participants who had greater exposure to long-term sleep loss to still suffer from sleep problems because their circadian rhythm might have been disrupted. With a mean PSQI of 4, we concluded that the majority of participants was able to adapt to normal sleeping behavior after years of exposure to sleep loss. However, $34 \%$ of participants scored above the cut-off for sleep problems; this did not correlate with the amount of exposure to, or recovery from sleep loss. Rather, this high percentage is common in studies on older adults, reporting that $16-34 \%$ of participants without any subjective sleep complaints have a high PSQI [15]. From the sleep-wake diary entries, participants showed regular and normal sleep with little variation, which matched our PSQI findings. Current sleep quality did not seem to be affected by previous exposure to work-related sleep loss, indicating that it is possible to recover from periods of sleep loss.

Additionally to our primary aim, we explored the effect of long-term work-related sleep loss on QoL and mood. All participants reported having a good QoL. Older participants indicated more problems concerning mobility, daily activities, and pain. Older age also indicated a lower utility score, which implies a poorer QoL and more daily complications. However, no correlation was found between the number of working years and QoL. Furthermore, none of the participants reported anxiety or depression. Taking into account that maritime pilots are trained to be emotionally stable in dangerous situations, this finding could be a trait specific to our cohort. Interestingly, this trait remained stable after retirement.

In summary, we found that long-term exposure to work-related sleep loss had no effects on cognitive function, early dementia symptoms, QoL, or mood. This is in contrast with earlier epidemiological studies that identified sleep loss as risk factor for later-life dementia. These studies focused mainly on intrinsic sleep problems, whereas we focused on extrinsic sleep loss. It is possible that intrinsic causes of sleep loss drive the relationship with dementia. For example, sleep loss could be a very early symptom of a neurodegenerative disease that manifests 10-20 years later [1,2]. Alternatively, an intrinsic sleep disorder may be a manifestation of a disease that harbors an increased risk of dementia. These theories could explain why sleep loss due to an extrinsic cause may not lead to an increased dementia risk. Arguing against these theories are short-term studies on acute extrinsic sleep deprivation that have shown associations with mechanisms underlying dementia $[9,16]$. These studies suggest that sleep loss, regardless of its cause, is mechanistically linked to pathological processes that increase dementia risk. Our results may be explained by the hypothesis that, in the population we studied, the adverse effects of sleep loss during a work week could be counteracted by rest periods during their week off, when maritime pilots can recover. Our data might suggest that this recovery period of 6-7 consecutive nights is sufficient to reverse the possible detrimental effects of sleep loss on cognitive function and amyloid or tau accumulation. This hypothesis is supported by results from Balkin et al. [17], who proposed that 3 nights of $8 \mathrm{~h}$ of sleep are sufficient to restore cognitive functions after 7 nights of partial sleep deprivation.

\section{Limitations and Implications}

This study addressed an important question related to the potential of long-term sleep loss to significantly impact cognitive function. Study limitations were the short habitual sleep assessment duration (10 days), the small sample size $(n=50)$, and the absence of objective measures of sleep. However, through their historical work schedules, which we 
Thomas et al.: Long-Term Occupational Sleep Loss and Post-Retirement Cognitive Decline

used in a different study with the same cohort, we verified that their sleep was indeed impaired during work weeks [18]. Even though our sample was relatively small (approx. $10 \%$ of all retired maritime pilots in The Netherlands), we achieved a broad variation in age and geographical location, which should result in a good representation of the retired maritime pilot population. However, our study involved a highly restricted population of males in a particular work setting (maritime pilots) who likely developed an ability to adapt to irregular sleep hours and compensate for this during their rest weeks; this reduces the generalizability of our results. Furthermore, it is possible that only cognitively healthy participants responded to the call for participation. We did receive responses from 2 retired pilots with dementia via their spouses, which could indicate that this bias may be limited, but we were unable to systematically collect information about retired maritime pilots who suffer from cognitive complaints/dementia. Privacy regulations and ethical considerations limited our options to obtain this information. Therefore, an exact number of maritime pilots who have developed AD/dementia cannot be given in the framework of this study. Nevertheless, given the high prevalence of MCI and dementia in the normal aging population, and the much higher prevalence hypothesized in maritime pilots, it would require a strong selection bias to obtain a sample of $10 \%$ without any cognitive symptoms. Many people with $\mathrm{MCI}$ and mild-stage dementia participate in society, live independently, drive cars, use the Internet etc., and many are unaware of their diagnosis. This makes it unlikely that all participants with $\mathrm{MCI} /$ dementia would have been unable to respond to our request to participate. Interviews were performed at or near participants' homes, further reducing the obstacle to participate.

\section{Conclusion}

Overall, this sample of retired maritime pilots reported no cognitive complaints or early dementia signs. They reported good sleep, a general satisfaction with their QoL, and stable mood. We found no indications that $>25$ years of exposure to work-related sleep loss led to cognitive decline or early dementia symptoms. Our data furthermore suggest, that it is possible to adapt to a normal sleeping pattern after years of work-related sleep loss through sufficient and well-timed rest periods.

\section{Acknowledgements}

We would like to thank all participants for taking part in this study and the secretary of the Dutch Maritime Pilot Association for helping with recruitment of participants.

\section{Statement of Ethics}

The study was approved by the ethical institutional review board at the Radboud UMC (Region: ArnhemNijmegen; file No. 2018-4557), performed according to the rules of good clinical practice (GCP), and complies with the rules for human experimentation stated in the World Medical Association Declaration of Helsinki. Written informed consent was obtained from all participants.

\section{Disclosure Statement}

The authors have no conflicts of interest to declare. 


\section{Funding Sources}

This study was funded in parts by an ISAO grant (Internationale Stichting Alzheimer Onderzoek, grant No. 15040). The funders had no role in the design and conduct of the study; collection, management, analysis, or interpretation of the data; preparation, review, or approval of the manuscript; or decision to submit the manuscript for publication.

\section{Author Contributions}

J.T. was involved in designing the project, setting up the study, recruiting participants, acquisition of data, analyzing and interpretation of the data, and writing this manuscript. S.O. was a major contributor in interpretation of the data, revising the manuscript and conceiving the ideas. J.A.H.R.C. was a major contributor in designing the project, obtaining funding, setting up the study, and was involved in writing and revising the manuscript. All authors approved the final version of the manuscript.

\section{References}

1 Ju YE, McLeland JS, Toedebusch CD, Xiong C, Fagan AM, Duntley SP, et al. Sleep quality and preclinical Alzheimer disease. JAMA Neurol. 2013 May; 70(5):587-93.

2 Slats D, Claassen JA, Verbeek MM, Overeem S. Reciprocal interactions between sleep, circadian rhythms and Alzheimer's disease: focus on the role of hypocretin and melatonin. Ageing Res Rev. 2013 Jan;12(1):188-200.

3 Vitiello MV, Borson S. Sleep disturbances in patients with Alzheimer's disease: epidemiology, pathophysiology and treatment. CNS Drugs. 2001;15(10):777-96.

4 Ju YE, Lucey BP, Holtzman DM. Sleep and Alzheimer disease pathology-a bidirectional relationship. Nat Rev Neurol. 2014 Feb;10(2):115-9.

5 Tsapanou A, Gu Y, Manly J, Schupf N, Tang MX, Zimmerman M, et al. Daytime sleepiness and sleep inadequacy as risk factors for dementia. Dement Geriatr Cogn Disord Extra. 2015 Jul;5(2):286-95.

6 Jagust WJ, Mormino EC. Lifespan brain activity, $\beta$-amyloid, and Alzheimer's disease. Trends Cogn Sci. 2011 Nov; 15(11):520-6.

7 Kang D, Lim HK, Jung WS, Jeong JH, Kim TW, Han JH, et al. Sleep and Alzheimer's Disease. Sleep Med Res. 2015; 6(1):1-9.

8 Jessen NA, Munk AS, Lundgaard I, Nedergaard M. The glymphatic system: a beginner's guide. Neurochem Res. 2015 Dec;40(12):2583-99.

9 Ooms S, Overeem S, Besse K, Rikkert MO, Verbeek M, Claassen JA. Effect of 1 night of total sleep deprivation on cerebrospinal fluid $\beta$-amyloid 42 in healthy middle-aged men: a randomized clinical trial. JAMA Neurol. 2014 Aug; 71(8):971-7.

10 Wei M, Zhao B, Huo K, Deng Y, Shang S, Liu J, et al. Sleep Deprivation Induced Plasma Amyloid- $\beta$ Transport Disturbance in Healthy Young Adults. J Alzheimers Dis. 2017;57(3):899-906.

11 Shokri-Kojori E, Wang GJ, Wiers CE, Demiral SB, Guo M, Kim SW, et al. $\beta$-Amyloid accumulation in the human brain after one night of sleep deprivation. Proc Natl Acad Sci USA. 2018 Apr;115(17):4483-8.

12 Holth JK, Fritschi SK, Wang C, Pedersen NP, Cirrito JR, Mahan TE, et al. The sleep-wake cycle regulates brain interstitial fluid tau in mice and CSF tau in humans. Science. 2019;363:880-4.

13 Claassen JA. New cardiovascular targets to prevent late onset Alzheimer disease. Eur J Pharmacol. 2015 Sep; 763 Pt A:131-4.

14 Ponds R, Van Boxtel M, Jolles J. De Cognitive Failure Questionnaire als maat voor subjectief cognitief functioneren. Tijdschrift voor Neuropsychologie. 2006;1(2):37-45.

15 Vitiello MV, Larsen LH, Moe KE. Age-related sleep change: gender and estrogen effects on the subjectiveobjective sleep quality relationships of healthy, noncomplaining older men and women. J Psychosom Res. 2004 May;56(5):503-10.

16 Ju YS, Ooms SJ, Sutphen C, Macauley SL, Zangrilli MA, Jerome G, et al. Slow wave sleep disruption increases cerebrospinal fluid amyloid- $\beta$ levels. Brain. 2017 Aug;140(8):2104-11.

17 Balkin TJ, Rupp T, Picchioni D, Wesensten NJ. Sleep loss and sleepiness: current issues. Chest. 2008 Sep; 134(3):653-60.

18 Thomas J, Ooms S, Verbeek M, Booij J, Rijpkema M, Kessels RP, et al. Sleep-cognition Hypothesis in Maritime Pilots: what is the effect of long-term work-related poor sleep on cognition and amyloid accumulation in healthy middle-aged maritime pilots: methodology of a case-control study. BMJ Open. 2019 Jun;9(6):e026992. 